\title{
Rapid topography mapping of scalar fields: Large molecular clusters
}

\author{
Sachin D. Yeole, ${ }^{1,2}$ Rafael López, ${ }^{3}$ and Shridhar R. Gadre ${ }^{1, a)}$ \\ ${ }^{1}$ Department of Chemistry, IIT Kanpur, Kanpur 208016, India \\ ${ }^{2}$ Department of Chemistry, University of Pune, Pune 411007, India \\ ${ }^{3}$ Departamento de Química Física Aplicada, Universidad Autónoma de Madrid, E-28049 Madrid, Spain
}

(Received 8 May 2012; accepted 2 August 2012; published online 21 August 2012)

\begin{abstract}
An efficient and rapid algorithm for topography mapping of scalar fields, molecular electron density (MED) and molecular electrostatic potential (MESP) is presented. The highlight of the work is the use of fast function evaluation by Deformed-atoms-in-molecules (DAM) method. The DAM method provides very rapid as well as sufficiently accurate function and gradient evaluation. For mapping the topography of large systems, the molecular tailoring approach (MTA) is invoked. This new code is tested out for mapping the MED and MESP critical points (CP's) of small systems. It is further applied to large molecular clusters viz. $\left(\mathrm{H}_{2} \mathrm{O}\right)_{25},\left(\mathrm{C}_{6} \mathrm{H}_{6}\right)_{8}$ and also to a unit cell of valine crystal at MP2/6-31+G(d) level of theory. The completeness of the topography is checked by extensive search as well as applying the Poincaré-Hopf relation. The results obtained show that the DAM method in combination with MTA provides a rapid and efficient route for mapping the topography of large molecular systems. (C) 2012 American Institute of Physics. [http://dx.doi.org/10.1063/1.4746243]
\end{abstract}

\section{INTRODUCTION}

Studies on scalar fields such as molecular electron density (MED), electron momentum density (EMD), and molecular electrostatic potential (MESP) are witnessing growing interest for the last three decades. The basic reason for this is that much chemical information is buried in the wave function of a molecular system, which can be readily unearthed using these scalar fields. This chemical information includes features such as bonding, lone pairs, weak interactions, bond strengths, molecular structure, reactivity, etc. Also, the above mentioned scalar fields can be subjected to experimental measurements, thereby providing a direct check on the values obtained from the corresponding theoretical treatment. Topography mapping summarizes the basic information of a scalar field and involves locating and characterizing its critical points (CP's), isosurfaces, contours, gradient paths, etc. ${ }^{1-4}$ The $\mathrm{CP}$ of a three-dimensional scalar function $f\left(x_{1}, x_{2}, x_{3}\right)$ is defined as a point $\mathrm{P}$ at which the partial derivatives vanish, viz. $\left.\frac{\partial f}{\partial x_{i}}\right|_{p}=0$ for $i=1,2,3$. The topography of the scalar field of MED has been pioneered and popularized over the past forty years by Bader and co-workers. ${ }^{1-3}$ It has culminated into the celebrated atoms in molecules (AIM) theory. The AIM analysis of a molecule starts with the mapping of all the CP's followed by tracing the gradient paths, mapping out atomic basins, etc. The topography of EMD and MESP has been probed in detail by Gadre and co-workers. ${ }^{5,6}$ They have developed a package called INDPROP (Ref. 7) for locating and characterizing all the CP's of MED, EMD, and MESP for molecular system. A variety of chemical applications of the topography mapping of MESP were reported. ${ }^{8}$ More recently, use of MESP topography for studying the phenomenon of molecular recognition has also been proposed. ${ }^{9}$

\footnotetext{
a) Author to whom correspondence should be addressed. Electronic mail: gadre@iitk.ac.in.
}

There are a number of packages available, for mapping the topography and carrying out further AIM analysis of electron density. These include AIMPAC suite of programs, ${ }^{10(\mathrm{a})}$ BUBBLE, ${ }^{10(\mathrm{~b})}$ SADDLE, ${ }^{11(\mathrm{a})}$ OMEGA, ${ }^{11(\mathrm{~b})}$ to name a few. An algorithm based on eigenvector following method $^{12(a)}$ for locating MED CP's proposed by Popelier is implemented in program MORPHY. ${ }^{12(b)}$ There exist packages such as XD2006, ${ }^{13(a)}$ TOPXD (Ref. 13(b)) which use experimentally determined electron density for AIM analysis. There has been substantial interest in mapping MED and MESP topography of crystals by experimental as well as theoretical means. ${ }^{1,4,14-16}$ These include the works of Gatti and coworkers, ${ }^{14(\mathrm{a}), 14(\mathrm{~b})}$ Munshi et al. ${ }^{14(\mathrm{c})}$ and Ghermani et al. ${ }^{15(\mathrm{a})}$ to mention a few. In such investigations, MED and MESP are evaluated from experimental x-ray scattering data and further used for AIM analysis of the crystal.

Recently, a new method for mapping the complete topography of MED and MESP for large molecules, which does not require the evaluation of the scalar functions on a threedimensional grid, was proposed and tested out. ${ }^{17}$ This previously proposed algorithm is improved upon in the present work and coupled with the deformed atoms in molecules (DAM) method ${ }^{18-22}$ which permits rapid, yet sufficiently accurate, evaluation of MED and MESP. The newly developed automated code is applied to large cluster systems viz. $\left(\mathrm{H}_{2} \mathrm{O}\right)_{25},\left(\mathrm{C}_{6} \mathrm{H}_{6}\right)_{8}$, and a unit cell of valine crystal.

\section{METHODOLOGY}

\section{A. Deformed atoms in molecules method}

Few decades ago, Stewart ${ }^{23}$ proposed a method of expansions of the density in terms of radial factors times spherical harmonics. Such a method was also used by Hansen and Coppens ${ }^{24}$ for computing electrostatic potential and its derivatives. Later, Rico and López et al. ${ }^{18-22}$ proposed an 
efficient DAM procedure for evaluation of molecular scalar fields such as MED and MESP by partitioning into atomic contributions. However, the difference between the DAM and Coppens-Hansen method comes from two sources: the way in which the density is partitioned into pseudoatomic fragments and the functions chosen for representing the radial factors.

In Coppens' procedure, a fit of the electron density (by means of integration of the structure factor) is made excluding the core- or spherical-atoms from the Fourier summations. This procedure is very well-suited for densities coming from $\mathrm{x}$-ray data. On the other hand, the DAM method can be only applied to the density theoretically computed in a LCAO calculation (at any level of computation). In this case, the density is expressed as a linear combination of one- and two-center charge distributions (densities). Each one of pseudoatoms in DAM consists of all the distributions centered at its nucleus and a part of each two-center distributions implying one of the functions centered in that nucleus. Therefore, only the twocenter distributions are partitioned and, to carry out this partition, a least deformation criterion based on the infinite-order expansion of the long-range potential is employed. ${ }^{19}$

In the representation of the radial factors, Coppens et al. choose Slater functions, whereas in DAM method, piecewise defined functions are used. The range of $r$ in which the density takes values above a given accuracy threshold is decomposed in appropriate intervals of radii $\lambda_{i}$ (i.e., $\lambda_{i-1} \leq \mathrm{r} \leq \lambda_{i}$ ). For intervals such that the values of the density at the boundaries $\left(\lambda_{i-1}, \lambda_{i}\right)$ differ in one order of magnitude or above, the radial factors are fitted to products of polynomials times one exponential with a suitable exponent. For intervals in which the variation of the radial factor is smaller, only polynomials are taken. As it happens in Coppens' method, the integrals leading to the electrostatic potential and its derivatives are analytical also in DAM when taken, and their efficient calculation is straightforward as it has been previously shown. ${ }^{20(b)}$ Although left and right limits of derivatives at boundaries are slightly different, these differences have proved to be sufficiently small to be negligible for practical purposes.

The recursive algorithms used in DAM enable to achieve high order expansions in spherical harmonics which, combined with the notable flexibility in the fit of the radial factors, allow to attain sufficient accuracy at a moderate cost for the efficient search of critical points both for MED and MESP.

As commented, in DAM method, the MED is expressed as the sum of atom-centered densities (cf. Eq. (1)), where each atomic fragment, $\rho^{A}(\mathbf{r})$, is determined by least deformation criterion. These atomic fragments are expanded as products of spherical harmonics and radial factors as given in Eq. (2).

$$
\begin{gathered}
\rho(\mathbf{r})=\sum_{A} \rho^{A}(\mathbf{r}), \\
\rho^{A}\left(\mathbf{r}_{A}\right)=\sum_{l=0}^{l \max } \sum_{m=-1}^{l} z_{l}^{m}\left(\mathbf{r}_{A}\right) f_{l m}^{A}\left(r_{A}\right) .
\end{gathered}
$$

Here, $z_{l}^{m}(\mathbf{r})$ are spherical harmonics and $f_{l m}^{A}(r)$ are the corresponding radial factors. The radial factors are fitted to piecewise analytic functions and expansion is carried out over the length $l_{\max }$. Using this density, the corresponding electrostatic potential at point $\mathbf{r}$ can be obtained by Eq. (3).

$$
\begin{aligned}
& V(\mathbf{r}) \\
& =\sum_{A=1}^{N}\left\{\frac{Z_{A}}{r_{A}}-\sum_{1=0}^{l \max } \sum_{m=-1}^{l} z_{l}^{m}\left(\mathbf{r}_{A}\right)\left[\frac{Q_{l m}^{A}\left(r_{A}\right)}{r_{A}^{2 l+1}}+q_{l m}^{A}\left(r_{A}\right)\right]\right\} .
\end{aligned}
$$

Here, $Z$ is nuclear charge and $Q_{l m}$ and $q_{l m}$ are auxiliary functions. The process of evaluation of MESP is equivalent to atomic multipolar expansion for long range interactions although it has been shown to work quite well for short range interactions also. The performance of the method for evaluation of MED and MESP has been tested out on many systems such as water, nitrobenzene, etc. ${ }^{20,21}$

This DAM method of evaluating the MED and MESP has been implemented in the package DAMQT. ${ }^{22}$ The advantage of this method is that the calculation of function and its derivatives is very rapid and is dependent only on the expansion parameter $l_{\max }$. Considering this, the MED and MESP evaluated by DAM, along with the gradients, are employed in conjunction with the topography building code reported in Ref. 17.

\section{B. Topography mapping algorithm}

As demonstrated by the work of Popelier ${ }^{25}$ on homomorphism between bare nuclear potential (BNP) and MED and our earlier work, ${ }^{17} \mathrm{BNP}$ acts as a starting point for mapping the corresponding MED topography. MED topography is richer in number of CP's than that of BNP. BNP and MED both together define the MESP and hence, in general, the number of MESP CP's is much larger than those individually for MED and BNP. The BNP CP's are used as guess points for MED topography, along with the points generated by joining the nuclei which lie within a cutoff distance. For MESP topography, MED critical points and points obtained by generating a cube around the nuclei are used as guess points.

For large molecules, use of molecular tailoring approach (MTA) is made. ${ }^{26}$ MTA is a fragmentation based method which enables the $a b$ initio calculations on large molecules. The details of the molecular tailoring method are described in many research articles from our group (see Ref. 26). However, some details of MTA relevant to the present study are as follows. Firstly the supermolecule is scissored into smaller parts termed as fragments on which a $b$ initio calculations are done. The density matrices (DM's) of fragments thus obtained are patched together to generate an accurate estimate of the DM of the supermolecule. ${ }^{26(b)}$ This MTA DM is further employed for evaluation of MED and MESP, which are subsequently used for topographical analysis.

In the current work, the topography of individual smaller fragments is mapped using the above mentioned procedure. The CP's generated for all the fragments are collectively given as guess points for mapping the topography of the supermolecule under reference.

The details of this step-by-step topography mapping algorithm are discussed in Ref. 17. In that work, INDPROP was used for evaluation of MED and MESP. In the present work, the DAM method described above is used for rapid 
evaluation of MED and MESP employing Eq. (2) and (3), and their derivatives, over the expansion length of $l_{\max }=15$. For this value of $l_{\max }$, the function values are found to match quite well their counterparts calculated by GAUSSIAN (Ref. 27) package. Also in the previous algorithm, the subroutine STEPIT (Ref. 28) was used for function minimization, while in the current code, L-BFGS, ${ }^{29}$ a gradient-based optimizer is employed. The topography mapping code is written in FORTRAN 90 and compiled using Intel Fortran compiler on Linux platform. In the line with our earlier works on parallelization of quantum chemical algorithms, ${ }^{30}$ the topography code is made parallel. Parallelization is implemented in the code by distributing the number of guess points on different processors and due to this, the time required for topography mapping is even smaller. Currently, the parallelization is limited to the one CPU with multiple cores. The implementation using more than one CPU is underway. Given the DM of the reference molecule, topography mapping can be carried out at any desired level of theory. The DM of large system at high level of theory is obtained using MTA. The completeness of the topography of a non-negative valued scalar field such as MED is checked by Poincaré-Hopf (PH) relation (see Eq. (4)), i.e., the Euler characteristic $\chi$ attains the value of -1 . In Eq. (4), $n_{+3}$ is the number of $(3,+3)$ CP's, $n_{+1}$ is the number of $(3,+1)$ CP's, and so on. For MESP, the PH relation takes the form of Eq. (5) wherein $n_{-}$and $n_{+}$denote the number of asymptotic maxima and minima, respectively. ${ }^{31}$

$$
\begin{gathered}
\chi=n_{+3}-n_{+1}+n_{-1}-n_{-3}=-1, \\
\chi=n_{+3}-n_{+1}+n_{-1}-n_{-3}=n_{-}-n_{+} .
\end{gathered}
$$

It may be re-emphasized that fulfillment of $\mathrm{PH}$ relation is a necessary (though not sufficient) condition for ensuring completeness of the $\mathrm{CP}$ search. The newly developed topography code is tested out on some test cases and further applied
TABLE I. The molecular electron density and molecular electrostatic potential critical points (CP's) obtained for the test systems at B3LYP/6-31+G(d) level of theory. $n_{+3}, n_{+1}, n_{-1}, n_{-3}$ denote the number of different types of

\begin{tabular}{|c|c|c|c|c|c|c|}
\hline Test system & $n_{+3}$ & $n_{+1}$ & $n_{-1}$ & $n_{-3}$ & $\chi$ & Time/min \\
\hline \multicolumn{7}{|c|}{ MED } \\
\hline LSD & 0 & 8 & 56 & 49 & -1 & 4 \\
\hline Zidovudine & 0 & 9 & 55 & 47 & -1 & 5 \\
\hline$(\mathrm{ZnS})_{10}$ & 1 & 12 & 30 & 20 & -1 & 3 \\
\hline \multicolumn{7}{|c|}{ MESP } \\
\hline LSD & 11 & 18 & 56 & 49 & 0 & 14 \\
\hline Zidovudine & 14 & 20 & 53 & 47 & 0 & 13 \\
\hline$(\mathrm{ZnS})_{10}$ & 11 & 27 & 36 & 20 & 0 & 10 \\
\hline
\end{tabular}
CP's and $\chi$ is the corresponding Euler characteristic. See text for details.

to large systems such as $\left(\mathrm{H}_{2} \mathrm{O}\right)_{25},\left(\mathrm{C}_{6} \mathrm{H}_{6}\right)_{8}$ and a unit cell of valine crystal at MP2/6-31+G(d) level of theory.

\section{RESULTS AND DISCUSSION}

\section{A. Test cases of small molecules}

First the rapid topography mapping code is appraised by testing it out on some relatively small systems, viz. lysergic acid diethylamide (LSD) and zidovudine drug molecules, and $(\mathrm{ZnS})_{10}$ cluster at B3LYP/6-31+G(d) level of theory. Topography of MED and MESP is mapped using the current method for these molecules. The numbers and types of MED and MESP CP's obtained are reported in Table I along with the wall-clock time required for topography calculation. All the timings in this article are measured on one Intel Quad Core machine with 8GB RAM. The numbers of CP's obtained satisfy the PH relation for MED topography (cf. Eq. (4)). For MESP topography, the PH relation is applied by finding out the number of asymptotic maxima and minima and it is seen that the relation in Eq. (5) holds true for all the systems.

The MED and MESP CP's for the test systems are depicted $^{32}$ in Figure 1. LSD molecule possesses $8(3,+1)$

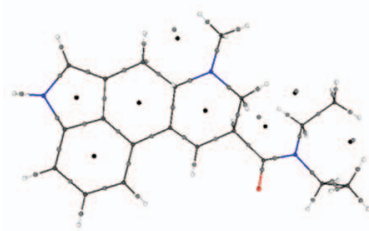

(a)

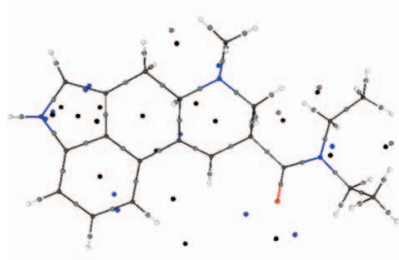

(a)

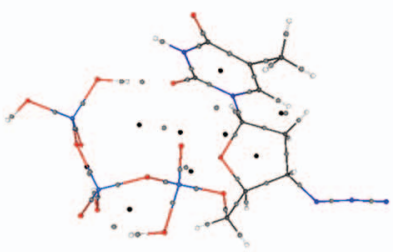

(b)

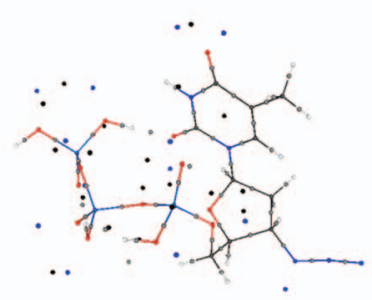

(b)

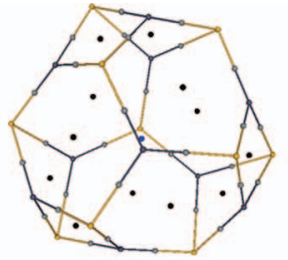

(c)

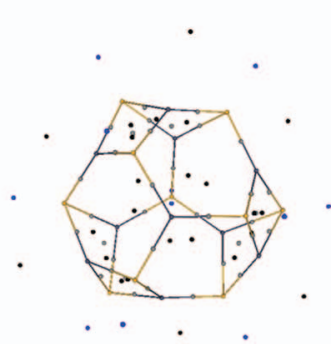

(c)

FIG. 1. Molecular electron density (MED) and electrostatic potential (MESP) critical points (CP's) for (a) LSD, (b) zidovudine, and (c) (ZnS) 10 cluster at B3LYP/6-31+G(d) level of theory. The blue dots denoting the $(3,+3)$ CP's, black dots denote the $(3,+1)$ CP's, and the gray dots the $(3,-1)$ CP's. See text for details. 


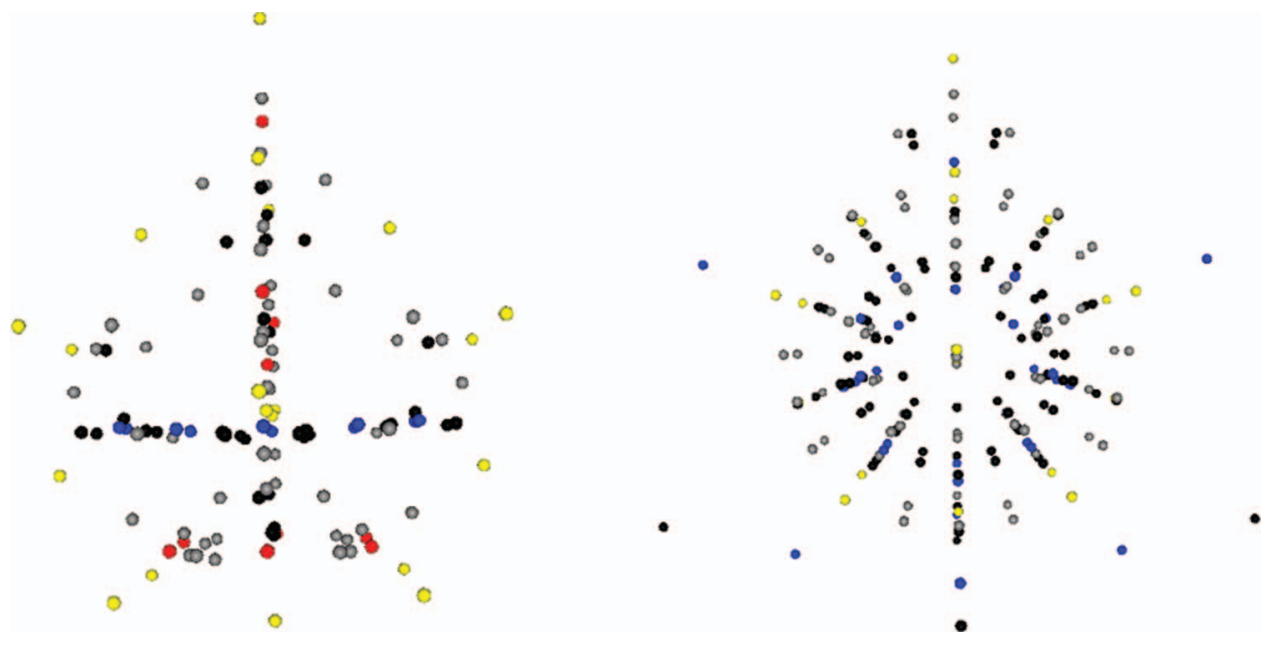

FIG. 2. Molecular electron density (MED) (left) and electrostatic potential (MESP) (right) critical points (CP's) for (Li) 20 cluster at MP2/6-31+G(d) level of theory. Notations are identical to those used in Figure 1. Red dots denotes the $(3,-3) \mathrm{CP}$ 's and yellow ones are Li nuclei. See text for details.

CP's out of which 4 represent the four rings and remaining four are due to H...H interactions. Zidovudine also has 9 (3, $+1)$ CP's out of which two are ring CP's and four represent the rings which are formed due to the H-bonding. The MED distribution of $\mathrm{ZnS}$ cluster exhibits one $(3,+3)$ cage minimum. The $12(3,+1)$ arise from the tetra- and hexa-atomic rings of $(\mathrm{ZnS})_{10}$ cage. The negative-valued $(3,+3)$ MESP minima of this cluster act as harbingers for the incoming electropositive $\mathrm{Zn}$ atom.

It is worthwhile pointing out a practical use of MESP topography for building up a given molecular crystal. There are three types of prominent negative-valued MESP minima present in topography of $(\mathrm{ZnS})_{10}$ of values -0.02713 , -0.02333 , and -0.01566 a.u. First incoming electropositive moiety may attack near most negative minimum $(-0.02713$ a.u.) and the possible site for the second one is the MESP minimum with a value of -0.02333 a.u. This feature is exploited for growing the cluster systematically, as described in the cluster building methodology detailed out in Ref. 33.

It has been proved earlier that the scalar field of MESP cannot have non-nuclear maxima. ${ }^{34}$ But MED is known to exhibit non-nuclear maxima in some cases. ${ }^{35,36}$ Gatti and co-workers have demonstrated the existence of non-nuclear maxima in the MED distributions of Li clusters. ${ }^{35}$ Considering this, the topography code is applied to map the MED and MESP CP's of a cluster containing 20 Lithium atoms at MP2/6-31+G(d) level of theory. The MED and MESP CP's of $(\mathrm{Li})_{20}$ are displayed in Figure 2. For this cluster system, 10 non-nuclear maxima are found in MED. There are also 10 (3, +3) minima and $33(3,+1)$ CP's found in the MED distribution. The MESP topography of this cluster system is even more rich containing total of 210 CP's, of which 33 are the (3, +3 ) minima. Here also the number of CP's obtained for MED and MESP found to satisfy respective $\mathrm{PH}$ relations.

\section{B. Larger cluster systems}

Having successfully demonstrated the accuracy and efficacy of our code for small test systems, the code is applied for mapping the topography of $\left(\mathrm{H}_{2} \mathrm{O}\right)_{25},\left(\mathrm{C}_{6} \mathrm{H}_{6}\right)_{8}$ and unit cell of valine crystal at MP2/6-31+G(d) level of theory. The CP's of these cluster systems are mapped following the hierarchy of BNP, MED and MESP following our earlier study. ${ }^{17}$ For such large test cases, the use of MTA is made for capturing the CP's. The whole cluster is scissored into smaller fragments and topography is mapped for such individual fragments. The CP's of all the fragments are used as guess points to obtain the CP's for whole cluster. The number of MED and MESP CP's obtained are displayed in Table II, along with the time required for this calculation. The MED CP's of these systems are shown in Figure 3. The number of MESP CP's being very large, only the $(3,+3)$ minima for these clusters are displayed $^{32}$ in Figure 4.

For $\left(\mathrm{H}_{2} \mathrm{O}\right)_{25}$ cluster, there are $5(3,+3)$ minima present in MED distribution, which are due to the cages formed by the five membered rings, as can be noticed from Figure 3(a). For each five membered ring formed by the H-bonding between water molecules, there is a $(3,+1) \mathrm{CP}$ along with some connecting $(3,+1)$ CP's. The MESP topography of this system shows a large number of CP's out of which 61 are (3, +3 ) minima. These minima are present outside as well as inside the cages. The location and MESP values at these minima could be used for positioning and studying the chemistry of small molecules inside the water cages. For benzene

TABLE II. The molecular electron density and molecular electrostatic potential critical points obtained for the test systems at MP2/6-31+G(d) level of theory. Notations are identical to those used in Table I. See text for details.

\begin{tabular}{lcrrrrr}
\hline \hline Test system & $n_{+3}$ & $n_{+1}$ & $n_{-1}$ & $n_{-3}$ & $\chi$ & Time/min \\
\hline & & & MED & & & \\
$\left(\mathrm{H}_{2} \mathrm{O}\right)_{25}$ & 5 & 27 & 96 & 75 & -1 & 12 \\
$\left(\mathrm{C}_{6} \mathrm{H}_{6}\right)_{8}$ & 9 & 34 & 120 & 96 & -1 & 12 \\
Valine & 18 & 73 & 206 & 152 & -1 & 13 \\
& & & MESP & & & \\
$\left(\mathrm{H}_{2} \mathrm{O}\right)_{25}$ & 61 & 119 & 133 & 75 & 0 & 31 \\
$\left(\mathrm{C}_{6} \mathrm{H}_{6}\right)_{8}$ & 24 & 58 & 129 & 96 & -1 & 32 \\
Valine & 28 & 84 & 209 & 152 & 1 & 42 \\
\hline \hline
\end{tabular}




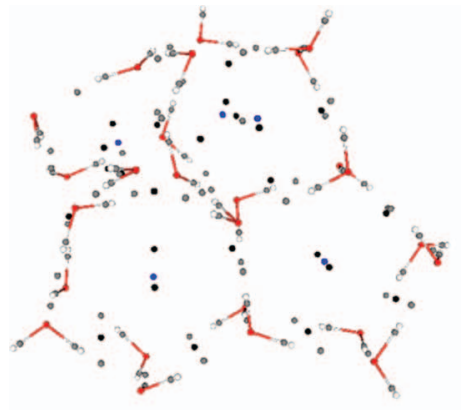

(a)

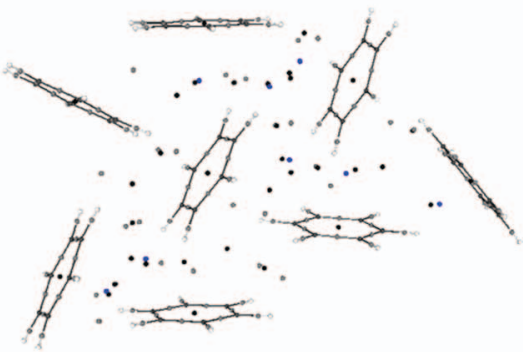

(b)

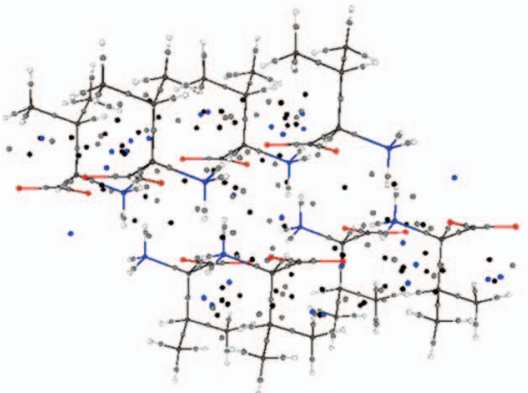

(c)

FIG. 3. Molecular electron density (MED) critical points (CP's) for (a) $\left(\mathrm{H}_{2} \mathrm{O}\right)_{25}$, (b) $\left(\mathrm{C}_{6} \mathrm{H}_{6}\right)_{8}$, and (c) unit cell of valine crystal at MP2/6-31+G(d) level of theory. Notations are identical to those used in Figure 1. See text for details.

cluster, there is a MED $(3,+3)$ minimum present at each contact point of three benzene rings. In MESP distribution, several CP's of isolated benzene rings are conspicuous by their absence as well as displaced in the benzene cluster. The (3, +3) minima present outside the cluster (cf. Figure 4(b)) are the probable positions of sites for metal binding and for new benzene monomer addition.

The valine crystal unit cell exhibits large network of MED CP's and the number goes even higher in the case of MESP. Though there are no rings in the system, there are

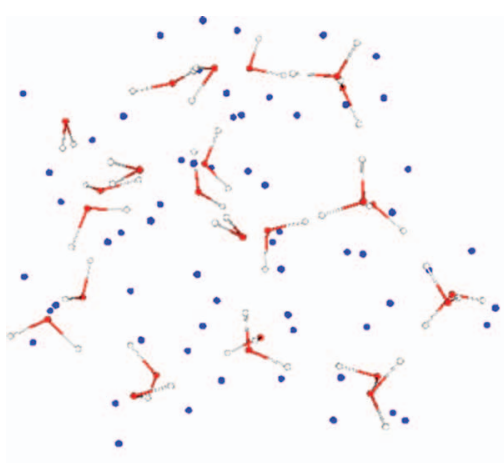

(a)

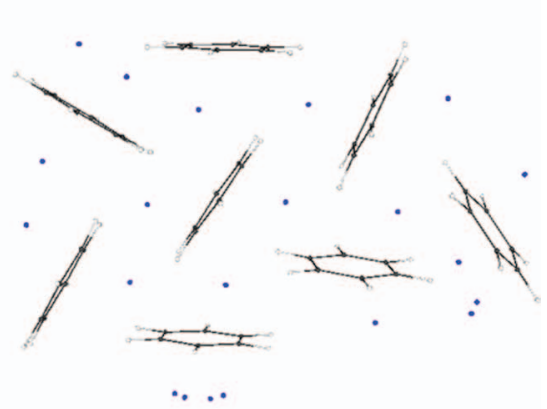

(b)

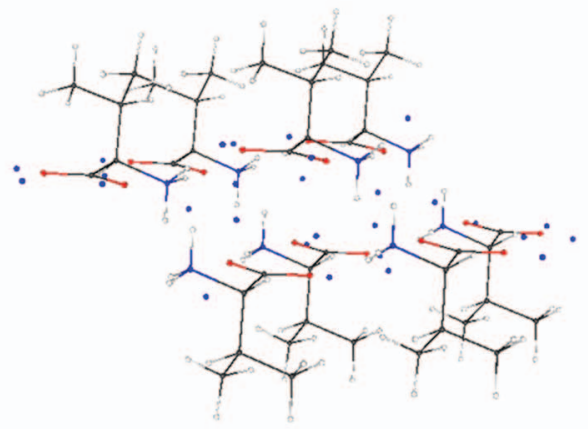

(c)

FIG. 4. Molecular electrostatic potential (MESP) $(3,+3)$ critical points $(\mathrm{CP}$ 's $)$ for $(\mathrm{a})\left(\mathrm{H}_{2} \mathrm{O}\right)_{25}$, (b) $\left(\mathrm{C}_{6} \mathrm{H}_{6}\right)_{8}$, and (c) unit cell of valine crystal at MP2/6-31+G(d) level of theory. Notations are identical to those used in Figure 1. See text for details. 
many $(3,+1)$ CP's present which connect pairs of $(3,+3)$ minima. All the MESP minima are seen to lie in middle of the crystal because of the presence $\mathrm{O}$ and $\mathrm{N}$ atoms. This shows that the monomer units are held together due to $\mathrm{O} \ldots \mathrm{H}$ and N...H interactions. From this, one can say that the MESP minima lying outside the unit cell (cf. Figure 4(c)) indicate the position where the valine monomer unit can be added to grow the crystal. The time required for topography mapping of MESP for this system is very small, viz. $42 \mathrm{~min}$ on a single quad core machine (as detailed above) when one considers the size of the unit cell (152 atoms and 1328 basis functions) at MP2 level of theory. At this high level of theory, even the DM calculation of the system is not possible by conventional actual calculation by GAUSSIAN or any other $a b$ initio software. This is because of the huge memory and disk space requirements of this calculation at MP2 level. But this task is made possible by the use of MTA wherein the MTA DM synthesized from the corresponding fragments DM's, is used for topography calculation.

\section{CONCLUDING REMARKS}

Topography mapping of scalar fields of molecular systems is of inherent importance, since it provides basic information about the molecular structure, bonding, reactive sites, etc. Locating and characterizing all CP's of a scalar field is an important part of the acclaimed AIM theory, proposed and developed by Bader and co-workers. ${ }^{1-3}$ The MED CP's serve as starting points for tracing the gradient paths for a given system. There are several methods available for mapping the CP's of MED and MESP in literature. Most of them involve an expensive step of evaluation of the corresponding function on a three-dimensional grid. For large systems, evaluation of function on a grid at high level of theory is indeed a formidable task. The present article has described a route for mapping the CP's of MED and MESP at any desired level of theory and basis set for large molecules. This has been demonstrated at B3LYP and MP2 levels of theory with a moderate size basis set as $6-31+\mathrm{G}(\mathrm{d})$. This is done considering the fact that the nature of scalar field of MED and MESP is independent of the level of theory and basis set, if a sufficiently large basis (incorporating diffuse/polarization functions) is employed. ${ }^{6(b)}$ The hierarchy of scalar fields BNP, MED and MESP is used for building the topography. ${ }^{13}$ DAM method is used for evaluation MED and MESP and respective gradients at guess point during optimization. The DAM method, based on partitioning of scalar field into atomic contributions, provides a rapid and sufficiently accurate function and gradient calculation.

This DAM method has been coupled with the previously described topography building algorithm. ${ }^{17}$ In the case of large molecules, MTA is employed for obtaining DM at high level of theory. The topography code has been applied to a variety of systems viz. H-bonding water cluster, a van der Waals cluster, $\left(\mathrm{C}_{6} \mathrm{H}_{6}\right)_{8}$ and a unit cell of valine crystal at MP2 level of theory. The code has been able to find the non-nuclear maxima for the classical test system of Li cluster. The completeness of the topography is checked by applying the corresponding $\mathrm{PH}$ relation and finding out the value of $\chi$, the
Euler characteristic. The computer time (on a Quad Core machine) required for largest system valine unit cell is $42 \mathrm{~min}$ for MESP topography calculation, which is very small as compared to the time required for MESP evaluation $(\sim 123 \mathrm{~min})$ on a grid of $101 \times 101 \times 101$ points using GAUSSIAN on the same hardware. However, this function evaluation on grid just provides probable starting guesses for CP's that needs further processing for actually locating and characterizing the CP's. With this experience of valine unit cell, one can hope that the topography code opens up the road for mapping the topography of large crystals.

The MED and MESP topography of molecular clusters reported in this work goes beyond the isolated molecule since they incorporate the effects of intermolecular interactions. It is hoped that the present work will provide an impetus to experimental investigations on molecular crystals.

\section{ACKNOWLEDGMENTS}

Authors thank the Center for Development of Advanced Computing (C-DAC), Pune for financial and computational support. S.R.G. is grateful to the Department of Science and Technology (DST) for the award of J. C. Bose National Fellowship. R. López acknowledges partial funding from the CAM (S2009_PPQ-1545, LIQUORGAS) and MICINN (CTQ2010-19232). Authors are also thankful to Dr. Graeme M. Day, University of Cambridge, for providing the coordinates of unit cell of valine crystal and to Dr. V. Subramanian, CLRI, Chennai for providing some test runs.

${ }^{1}$ R. F. W. Bader, Atoms in Molecules: A Quantum Theory (Clarendon, Oxford, 1990).

${ }^{2}$ R. F. W. Bader, T. T. Nguyen-Dang, and Y. Tal, J. Chem. Phys. 70, 4316 (1976); Y. Tal, R. F. W. Bader, and J. Erkku, Phys. Rev. A 21, 1 (1980).

${ }^{3}$ N. O. Malcolm and P. L. A. Popelier, J. Comput. Chem. 24, 437 (2003).

${ }^{4}$ S. R. Gadre and R. N. Shirsat, Electrostatics of Atoms and Molecules (Universities Press, Hyderabad, 2000).

${ }^{5}$ S. A. Kulkarni, S. R. Gadre, and R. K. Pathak, Phys. Rev. A 45, 4399 (1992); S. A. Kulkarni and S. R. Gadre, Z. Naturforsch. A 48, 145 (1993); P. Balanarayan and S. R. Gadre, J. Am. Chem. Soc. 128, 10702 (2006).

${ }^{6}$ S. R. Gadre, S. A. Kulkarni, and I. H. Shrivastava, J. Chem. Phys. 96, 5253 (1992); S. R. Gadre, S. A. Kulkarni, C. H. Suresh, and I. H. Shrivastava, Chem. Phys. Lett. 239, 273 (1995).

${ }^{7}$ INDPROP, the molecular property calculation package developed at Theoretical Chemistry Group, Department of Chemistry, University of Pune, Pune, India. See S. V. Bapat, R. N. Shirsat, and S. R. Gadre, Chem. Phys. Lett. 200, 373 (1992).

${ }^{8}$ S. R. Gadre and C. H. Suresh, J. Org. Chem. 62, 2625 (1997); S. R. Gadre and S. S. Pundlik, J. Phys. Chem. B 101, 3298 (1997); C. H. Suresh and S. R. Gadre, J. Phys. Chem. A 111, 710 (2007); G. Mehta, F. A. Khan, S. R. Gadre, R. N. Shirsat, B. Ganguly, and J. Chandrasekhar, Angew. Chem., Int. Ed. Engl. 33, 1390 (1994).

${ }^{9}$ D. K. Roy, P. Balanarayan, and S. R. Gadre, J. Chem. Sci. 121, 815 (2009).

${ }^{10}$ T. A. Keith and T. K. Gristmill, AIMAll, version 11.06.19, Software, Overland Park KS, USA, 2011; P. Krug, Program BUBBLE, McMaster University, 1990.

${ }^{11}$ K. Laidig, Program SADDLE, McMaster University, 1989; F. W. Biegler-König, T. T. Nguyen-Dang, Y. Tal, R. F. W. Bader, and A. J. Duke, J. Phys. B: At., Mol. Opt. Phys. 14, 2739 (1981).

${ }^{12}$ P. L. A. Popelier, Comput. Chem. Phys. Lett. 228, 160 (1994); Comput. Phys. Commun. 93, 212 (1995).

${ }^{13}$ A. Volkov, P. Macchi, L. J. Farrugia, C. Gatti, P. Mallinson, T. Richter, and T. Koritsanszky, XD2006 Program, 2006; A. Volkov, C. Gatti, Y. Abramov, and P. Coppens, TOPXD Program. Acta Cryst. A 56, 252 (2000). 
${ }^{14}$ C. Gatti, V. R. Saunders, and C. Roetti, J. Chem. Phys. 101, 10686 (1994); Modern Charge-Density Analysis edited by C. Gatti and P. Macchi (Springer, Heidelberg, 2012); P. Munshi, C. Jelsch, V. R. Hathwar, and T. N. Guru Row, Cryst. Growth Des. 10, 1516 (2010).

${ }^{15}$ N. E. Ghermani, C. Lecomte, and Y. Dusausoy, Phys. Rev. B 53, 5231 (1996); P. Coppens, Y. Abramov, M. Carducci, B. Korjov, I. Novozhilova, C. Alhambra, and M. R. Pressprich, J. Am. Chem. Soc. 121, 2585 (1999); R. Flacau, S. Desgreniers, and J. S. Tse, J. Chem. Phys. 129, 244507 (2008).

${ }^{16}$ C. Lecomte, M. Souhassou, and S. Pillet, J. Mol. Struct. 647, 53 (2003); B. Guillot, N. Muzet, E. Artacho, C. Lecomte, and C. Jelsch, J. Phys. Chem. B 107, 9109 (2003).

${ }^{17}$ S. D. Yeole and S. R. Gadre, J. Phys. Chem. A 115, 12769 (2011).

${ }^{18}$ J. F. Rico, R. López, J. M. G. de la Vega, and J. I. F. Alonso, J. Mol. Struct.: THEOCHEM 120, 163 (1985); J. F. Rico, J. R. Á. Collado, and M. Paniagua, Mol. Phys. 56, 1145 (1985).

${ }^{19}$ J. F. Rico, R. López, and G. Ramírez, J. Chem. Phys. 110, 4213 (1999); 117, 533 (2002).

${ }^{20}$ J. F. Rico, R. López, I. Ema, G. Ramírez, and E. V. Ludeña, J. Comput. Chem. 25, 1355 (2004); J. F. Rico, R. López, I. Ema, and G. Ramírez, ibid. 25, 1347 (2004).

${ }^{21}$ J. F. Rico, R. López, I. Ema, and G. Ramírez, J. Mol. Struct.: THEOCHEM 727, 115 (2005).

${ }^{22}$ R. López, J. F. Rico, G. Ramírez, I. Ema, and D. Zorrilla, Comput. Phys. Commun. 180, 1654 (2009).

${ }^{23}$ R. F. Stewart, Chem. Phys. Lett. 65, 335 (1979).

${ }^{24}$ N. K. Hansen and P. Coppens, Acta Cryst. A 34, 909 (1978); A. Volkov, H. F. King, P. Coppens, and L. J. Farrugi, ibid. 62, 400 (2006).
${ }^{25}$ P. L. Popelier and E. A. Bremond, Int. J. Quantum Chem. 109, 2542 (2009).

${ }^{26}$ V. Ganesh, R. K. Dongare, P. Balanarayan, and S. R. Gadre, J. Chem. Phys. 125, 104109 (2006); K. Babu, V. Ganesh, S. R. Gadre, and N. E. Ghermani, Theor. Chem. Acc. 111, 255 (2004).

${ }^{27}$ M. J. Frisch, G. W. Trucks, H. B. Schlegel et al., GAUssian 09, Revision A. 1, Gaussian, Inc., Wallingford, CT, 2009.

${ }^{28}$ J. P. Chandler, The subroutine STEPIT, distributed by QCPE, University of Indiana, Bloomington, IN, USA, 1967.

${ }^{29}$ J. L. Morales and J. Nocedal, ACM Trans. Math. Softw. 23, 550 (2011).

${ }^{30}$ S. R. Gadre, S. A. Kulkarni, A. C. Limaye, and R. N. Shirsat, Z. Phys. D: At., Mol. Clusters 18, 357 (1991); R. N. Shirsat, A. C. Limaye, and S. R. Gadre, J. Comput. Chem. 14, 445 (1993); A. C. Limaye, and S. R. Gadre, J. Chem. Phys. 100, 1303 (1994).

${ }^{31}$ M. Leboeuf, A. M. Köster, K. Jug, and D. R. Salahub, J. Chem. Phys. 111, 4893 (1999); D. K. Roy, P. Balanarayan, and S. R. Gadre, ibid. 129, 174103 (2008).

${ }^{32}$ UNIVIS-2000, a comprehensive visualization package; see A. C. Limaye and S. R. Gadre, Curr. Sci. 80, 1296 (2001); The package MeTA Studio: V. Ganesh, J. Comput. Chem. 30, 661 (2009).

${ }^{33}$ S. D. Yeole and S. R. Gadre, J. Chem. Phys. 134, 084111 (2011).

${ }^{34}$ S. R. Gadre and R. K. Pathak, Proc. Indian Acad. Sci., Chem. Sci. 102, 189 (1990); J. Chem. Phys. 93, 1770 (1990).

${ }^{35}$ C. Gatti, P. Fantucci, and G. Pacchioni, Theor. Chim. Acta 72, 433 (1987).

${ }^{36}$ W. L. Cao, C. Gatti, P. J. McDougall, and R. F. W. Bader, Chem. Phys. Lett. 141, 380 (1987); J. Cioslowski, J. Phys. Chem. 94, 5496 (1990); K. B. Wiberg, R. F. W. Bader, and C. D. H. Lau, J. Am. Chem. Soc. 109, 985 (1987). 